\title{
Case for the improvement of suboptimal care of gout patients in Hawai'i
}

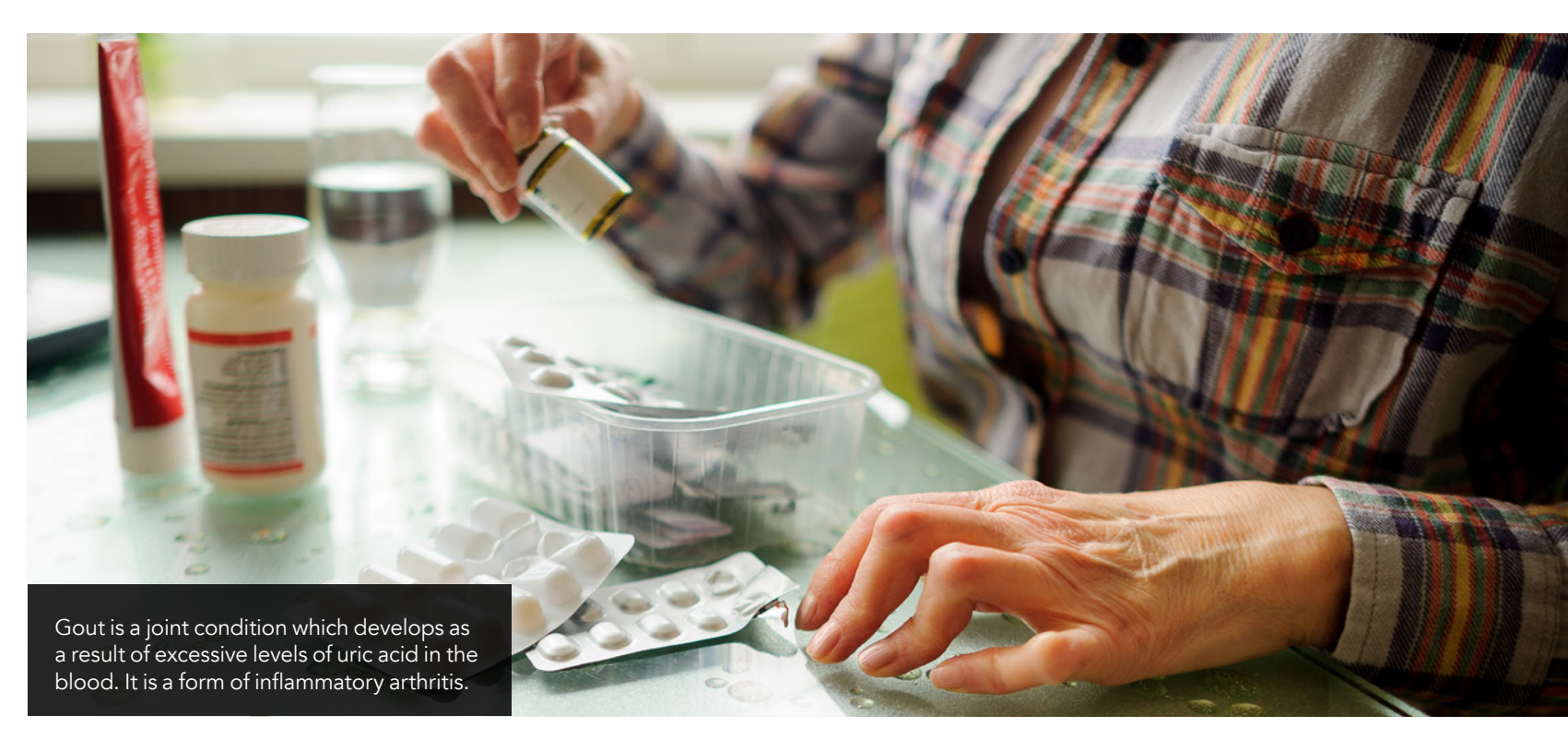

improvement of care for gout patients be put in place.

HIGHLIGHTS OF THE STUDY

Dr Lim and his colleagues did just that in their study in 2019. The study highlights the critical de in Hawai'i. Between 2011 and 2017 , gout patients visited the emergency department more often than rheumatoid arthritis patients, likely due to more episodes of gout flares arising from suboptimal care for gout patients. Near absence of data specific to the region presented significant challenges to the comparison of healthcare utilisation patterns for both groups. A retrospe chart review of both gout and RA patients was therefore undertaken. Drim was his co-authors had access Patients' medical records at Hawa'i' Pacific Health, which is one of the largest healthcare providers in Hawai't.

Included in the study were patients 18 (attended an outpatient setting, emergency department or were hospitalised) with any of the various facilities of Hawai'i Pacific Health healthcare system with a diagnosis of gout on discharge. They also must have had a follow-up data of at least one year at the time of the study. The RA group of patients was selected analysis of gout halthcore unistive patterns be made with that of a sil ar patterns be made with tha
The results revealed that between emergency department visits) with a primary discharge diagnosis of gout. In comparison, there were a total of 7272 encounters with a primary discharge diagnosis of RA within the same period.

Although the annual rates for emergency department visits and outpatient visits were trending lower and inpatient period for gout patients, the group department visits.

In contrast, RA patients had very low and stable admission rates during the study period, and most of the healthcare utilisation centred mainly on the outpatient setting with practically little or no emergency department visits.

The study also compared the costs of

When patient compliance with medication is combined with lifestyle changes, lower levels of uric acid can be achieved with the prospect of fewer gout flares.

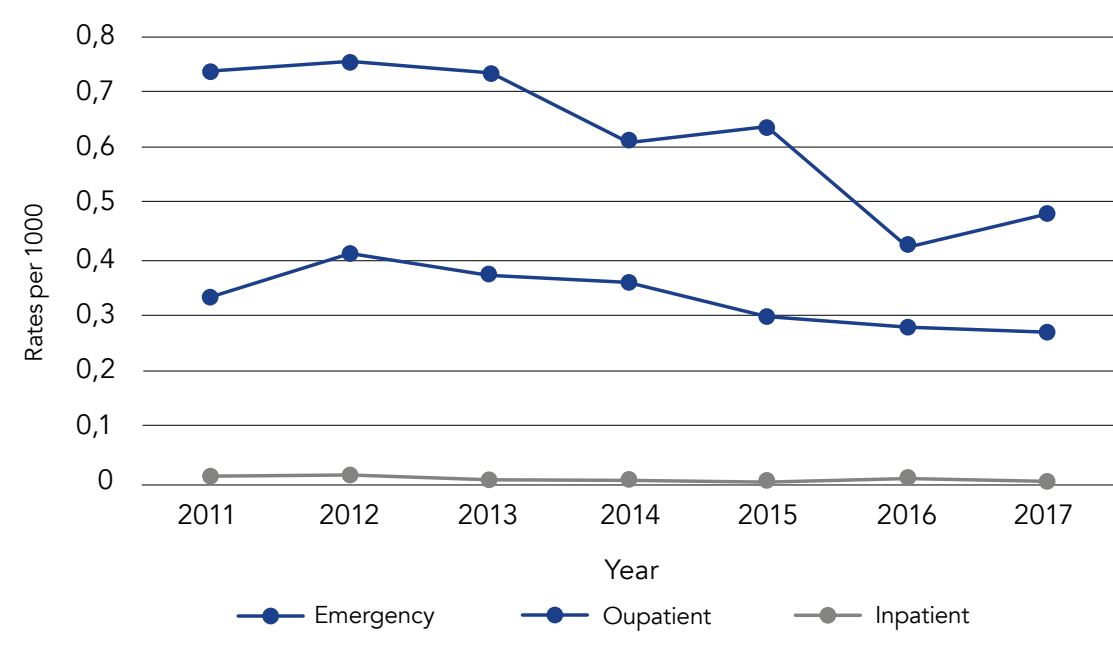

Fig 1. Annual rates of emergency department visits, outpatient visits and inpatient hospitalisations wit
a primary discharge diagnosis of gout $2011-2017$. (2011-2017 there were a total of 9552 


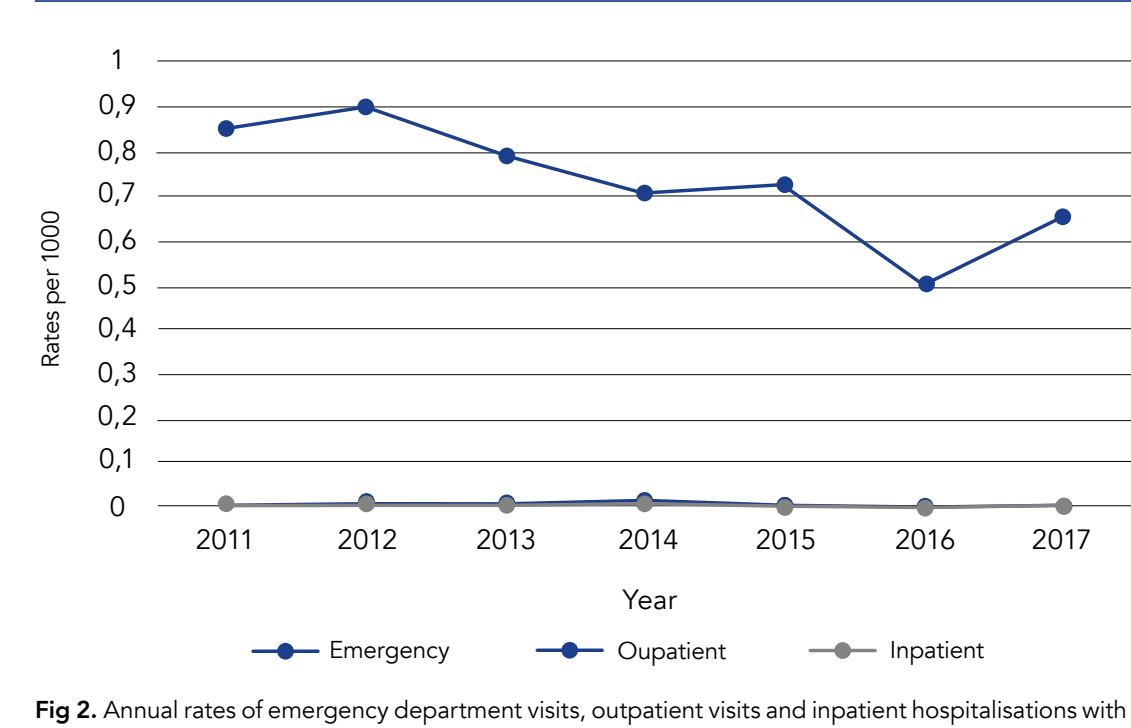

Fig 2. Annual rates of emergency department visits, outpatient
a primary discharge diagnosis of rheumatoid arthritis $2011-2017$

of gout patients with those of the RA group. The study revealed that in 2017 for example, the mean cost for every patient was approximately 3.4 times the cost of an outpatient visit In the RA group, an emergency department visit cost approximately 3.5 times the cost of an outpatient visit during the same year.

Although emergency department utilisation rates for gout patients declined between 2011 and 2017, the emergency department visits represented a

significant portion of healthcare utilisation for gout patients while RA patients were manage.d mainly in the outpatien vists outpatient visits, efforts must be made to reduce the healthcare costs of gout patients by facilitating the outpatient evaluation of gout patients and reducing emergency department utilisation.

RECOMMENDATIONS FOR IMPROVED CARE FOR GOUT Many emergency department visits

With increasing levels of adiposity, hypertension and high rates of diuretics utilisation worldwide, the prevalence of gout has increased significantly.
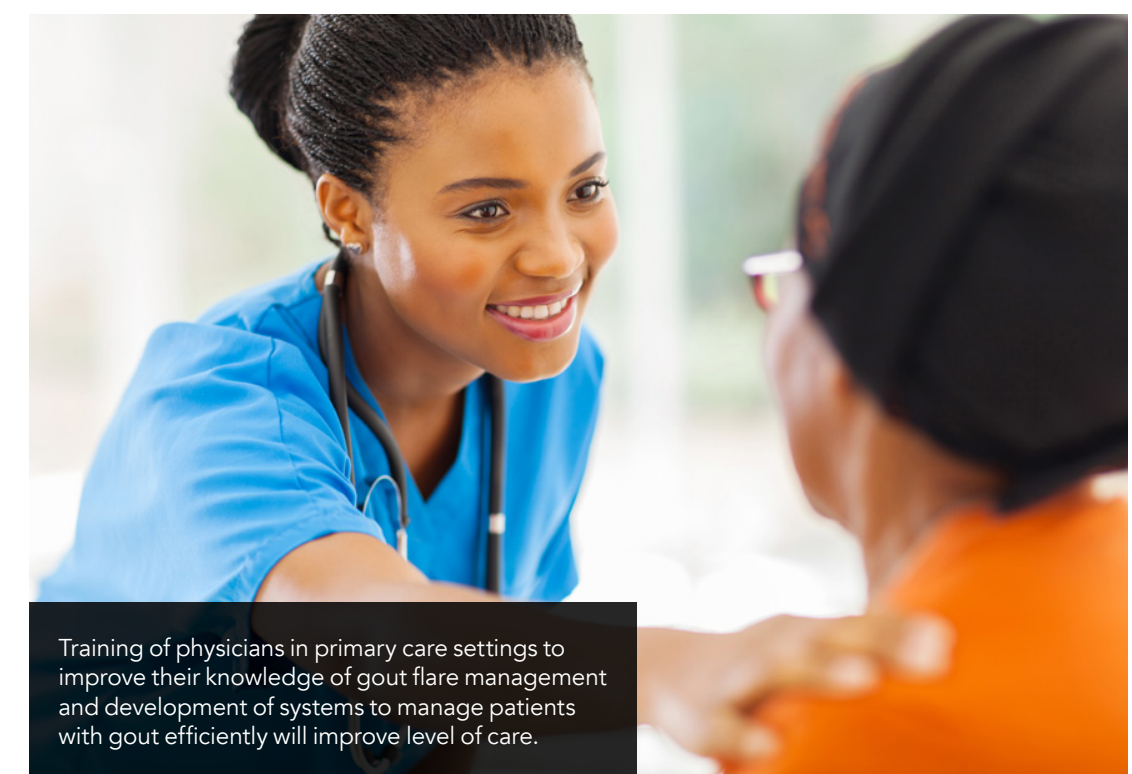

by gout patients can be prevented if gaps in the management of chronic care will go a long way in reducing emergency department visits, especially during a gout flare Establishment of urgent care clinics and extension of primary care operating hours will reduce unnecessary emergency department visits and hospitalisations.

Gout patients are often treated by primary care physicians. Training physicians in primary care settings to improve their knowledge of gout flare management and development of systems to manage patients with gout efficiently will improve level of care. In this regard, it is essential that healthcare providers manage gout strictly according tevels should be activ. Serum nic acid physicians to prevent gout flares.

Gout patients have their own role to play in cutting down the number of emergency department visits and reducing the cost to the healthcare system. Here, patient education is essential to improve medication compliance. Healthcare providers must engage with their patients and have discussions with them on lifestyle changes. Buttressing this fact, Dr Lara Pullen in an article in the online publication, The Rheumatologist, pointed out that these advice 'can include suggestions on obesity, alcoho use, fructose consumption and the This will helion of purine-rich fatty foods' lifestyle and cut down the burden of gout on the healthcare system. Whe patient compliance with medication is combined with lifestyle changes, lower levels of uric acid can be achieved with he prospect of fewer gout flares.

\section{CONCLUSION}

Gout patients in Hawai' have a high

number of emergency department

visits which is associated with

significant cost to the healthcare

system. As gout is a treatable disease, efforts should be made to implement the recommendations in this study of gout patients in Hawaili with a view to reducing the burd ith a view to reducing the by

\section{Behind the Research} Sian Yik Lim

E: limsianyik@gmail.com T: +1 808-486-6000 T: +1 808-485-4120

\section{Research Objectives}

Sian Yik Lim is a rheumatologist who has specia interests in osteoporosis, gout and ultrasound-guided joint injections.

\section{Detail}

Sian Yik Lim

Moanalua Road,

Medical Office Building, Suite 300

Aiea, HI 9670

Bio

Sian Yik Lim is a rheumatologist at the Hawai'i Pacific Health Bone \& Joint Center at Pali Momi Medical Center, where he takes care of many gout patients. His interests include clinical epidemiology where he is focused on patient orientated research of various conditions in Hawarí.

Funding
Hawai'i Pacific Health Summer Student Research Program

National Institute of Health grants U54MD007584, U54MD00760131, and 2U54GM104944-06

\section{Collaborators}

Victoria P. Mak

- So Yung Choi

- Hyeong Jun A

\section{References}

Lim, S, Lu, N, Oza, A, Fisher, M, Rai, S, Menedez, M, Choi, H. (2016) 'Trends in Gout and Rheumatoid Arthritis Hospitalisations in the United States', Journal of American Medical Association, 315 (21), 2345 -2347. https://www. ncbi.nlm.nih.gov/pmc/articles/PMC5064439/

Zhu, Y, Pandya, B, Choi, H. (2011) 'Prevalence of Gout and Hyperuricemia in the US General Population: The National Health and Nutrtion Examination Survey 2007

Pullen, L. (2016) 'Gout Treatment \& Care Remain Suboptimal' The Rheumatologist, available at https./ www.the-rheumatologist.org/article/gout-treatment-care remain-suboptimal//

\section{Personal Response}

What would be the modalities for ensuring that the findings and recommendations emanating
from this study are implemented by health authorities in Hawai'i

II Through our research, we hope to increase awareness about the burden of gout in Hawa'i By shining a light on increased emergency department visits due to gout, we hope that this will serve as a starting point for the health care systems in Hawail to address gout care going forward.

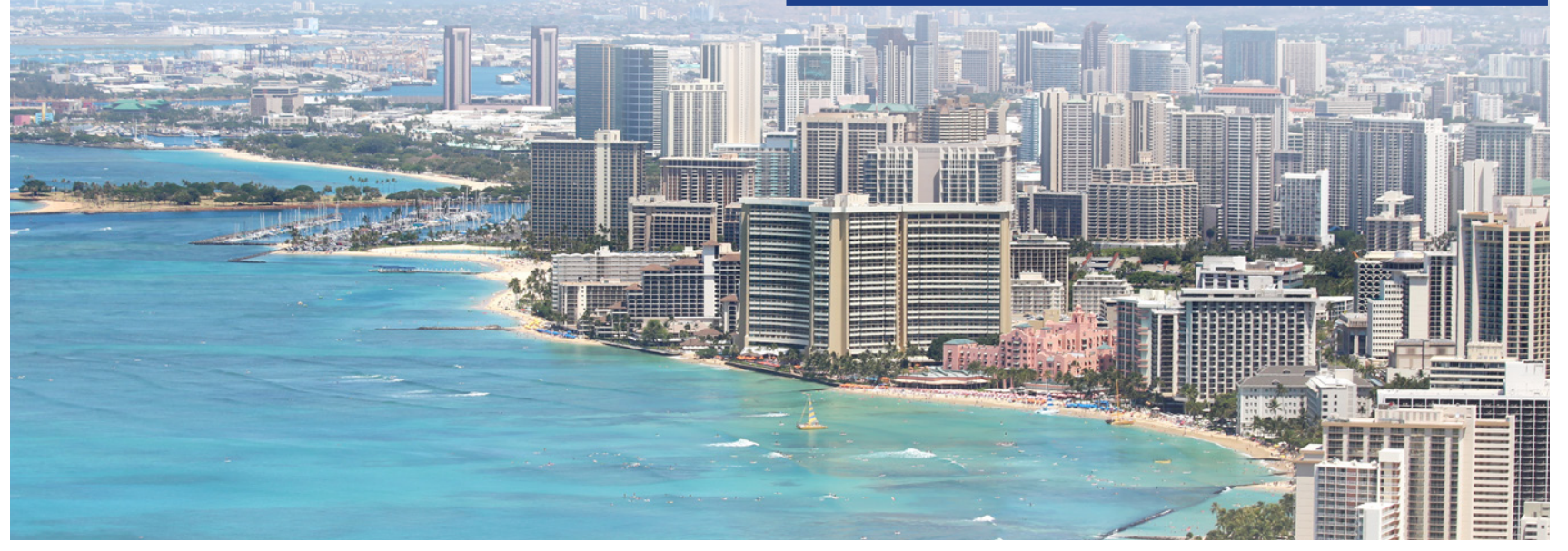

